\title{
Methods based on CNC grinding fault Distribution Parameter Estimation for Reliability Assessment
}

\author{
Jinwei Fan ${ }^{\mathrm{a}}$, Shi $\mathrm{Ji}^{\mathrm{b}}$, Hongliang Wang $^{\mathrm{c}}$, Lanqing Zhang ${ }^{\mathrm{d}}$ and Haohao Tao ${ }^{\mathrm{e}}$ \\ School of Mechanical Engineering, Beijing University of Technology, Beijing 100124, China. \\ ajwfan@bjut.edu.cn, b1469578848@qq.com, '977714868@qq.com ${ }^{\mathrm{d}} 1002878319 @ q q . c o m$ e87278 \\ 1344@qq.com
}

Keywords: Reliability, Probability density function, Least square method, Parameter estimation, MTBF, CNC grinding machine tools

\begin{abstract}
Fault distribution type identification and probability density function parameters estimation are the prerequisite of CNC grinding machine tools' reliability assessment. The procedure of fault data grouping, the technique of probability density function parameters estimation based on least square method and the method of reliability index( mean time between failures, MTBF) calculation are proposed. The probability density function parameters estimation and curve fitting, the comparison of goodness of fit, the MTBF solution of some type crankshaft are carried out by the method proposed in the paper. The results of statistical calculation and direct calculation by definition are close. The procedure of reliability assessment proposed here can be programmed easily and it provides one measure for reliability assessment of CNC grinding machine tools.
\end{abstract}

\section{Introduction}

With the technological innovation increasingly difficult situation, the competitiveness of products is the key to the level of reliability. Improve product reliability is built on the premise failure probability density function to determine the type of product failure distribution, to assess the current level of reliability of products. Reliability Assessment products at home and abroad to carry out a lot of research has made some achievements. Samanta B proposed the use of fault tree minimal cut-set method of reliability assessment ${ }^{[1]}$, this method requires the establishment of fault tree products, less efficient. Yang $\mathrm{Na}$ and others on the qualitative and quantitative methods of mechanical system reliability has been studied, but not for the calculation of reliability indicators elaborated $^{[2]}$. Literature ${ }^{[3-4]}$ using Monte Carlo simulation to evaluate the reliability of the product, and to assess the accuracy of the method depends on the a priori distribution function of the correctness of the calculation and large costs. Literature ${ }^{[5-6]}$ proposed the use of Bayesian networks to evaluate the reliability of products, the establishment of workload Bayesian network, and if you

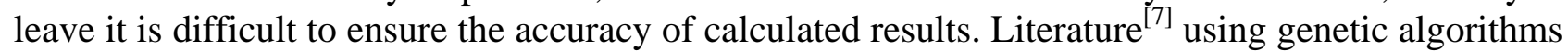
to solve the fault parameters of the distribution function. Currently, the study of the reliability evaluation method focused on aerospace and military equipment, relatively few studies on the general reliability of mechanical and electrical equipment. Based on this background, in terms of CNC grinding machine fault data statistics, common faults distribution type, various parameters estimation and distribution reliability indices calculation carried out research, the data processing from fault to fault distribution fitting and reliability the method of calculation. The method can easily form a computer program for evaluating the reliability of CNC grinding machines provide a convenient.

\section{Determine the type of fault distribution}

Fault Statistics.To find fault distribution of mechanical and electrical products, in order to assess the level of reliability of the product, must be do the product reliability test, collecting fault data. In order to increase the credibility of the statistical fault data, you must collect enough fault data, usually do the time censored reliability test. 
After taking the reliability data, the fault data are grouped according to time, the group size of number should be fit. Can uses the formula (1) to calculate the size of the number of groups ${ }^{[8]}$.

$$
\hat{k}=1+3.3 \log n_{T}
$$

Where: $n_{T}$ - The total number of faults within a certain censored time.

After calculating by following formula (1), Its rounding to obtain a packet $\mathrm{k}$. In order to ensure the accuracy of the fitting, if the calculated $\mathrm{k}$ value of less than 8,8 can be taken. After obtaining the $\mathrm{k}$ value, the censored time divided into $\mathrm{k}$ intervals, statistics of the number of fault each time, drawn into a table as shown in Table 1.

Tab.1 Grouping of Fault Data

\begin{tabular}{ccccc}
\hline No. & $t_{i-}$ & $t_{i+}$ & $t_{i}$ & $n_{i}$ \\
\hline 1 & 0 & $\mathrm{~T} / k$ & $\mathrm{~T} / 2 k$ & $n_{1}$ \\
2 & $\mathrm{~T} / k$ & $2 \mathrm{~T} / k$ & $3 \mathrm{~T} / 2 k$ & $n_{2}$ \\
$\ldots$ & $\ldots$ & $\ldots$ & $\cdots$ & $\cdots$ \\
$k$ & $(k-1) T / k$ & $T$ & & $n_{k}$ \\
\hline
\end{tabular}

Where: $t_{i-}$-packet interval left point; $t_{i+}$-packet interval right point; $t_{i}$-median packet interval; $n_{i}$ - The number of faults in the group i statistics; $T$ — censored time.

Determine the type of fault distribution.After obtaining the number of faults within each section can using formula (2) to obtain observations within each interval fault probability density.

$$
\hat{f}\left(t_{i}\right)=\frac{n_{i}}{n_{t} t}
$$

Where: $t$-section length

According to formula (2) the fault probability density of observations obtained can be plotted scattergram fault probability density observations. According to preliminary scatterplot since you can judge which products are distributed.

\section{Parameter Estimation}

Least squares method. In determining the initial distribution of fault type, fault parameters required to estimate the probability density function. Simply least squares method is one of useful parameter estimation method[9], the basic idea is assumed that there are n observations $\{x i, y\} \mathrm{i}(\mathrm{i}=$ $1,2, \ldots, n$ ), if there is a linear relationship between $\mathrm{x}$ and $\mathrm{y}$, then it can be used to fit a straight line relationship between the change in $\mathrm{x}$, $\mathrm{y}$ between the formula (3) below.

$$
\hat{y}=a \hat{x}+b
$$

Least squares method can be obtained formula (3) the parameters a and $b$ values such as formula (4) below.

$$
\left\{\begin{array}{l}
a=\frac{1}{n} \sum_{i=1}^{n} y_{i}-\frac{b}{n} \sum_{i=1}^{n} x_{i} \\
b=\frac{\sum_{i=1}^{n}\left(x_{i}-\frac{1}{n} \sum_{i=1}^{n} x_{i}\right)\left(y_{i}-\frac{1}{n} \sum_{i=1}^{n} y_{i}\right)}{\sum_{i=1}^{n}\left(x_{i}-\frac{1}{n} \sum_{i=1}^{n} x_{i}\right)}
\end{array}\right.
$$

After the fault to take advantage of the least squares method to estimate the parameters of the distribution function, we need to construct a linear relationship using the formula (4) to estimate the value of $a$ and $b$, the reverse probability density function parameters. So configured linear relationship is the key parameter estimation using least square method. Here for two kinds of CNC grinding the most common faults distribution to construct a linear relationship, the parameters of the failure distribution function thus estimated.

Exponential distribution. Expressions cumulative distribution function of index distribution of 
formula (5), there is an argument $\lambda$

$$
F(t)=1-e^{-\lambda t}
$$

Taking the logarithm of the formula (5)can get formula (6), can be obtained ,if the sample data that is an exponential distribution, then formula (6) satisfies a linear relationship, the parameter for the exponential distribution is $\lambda=-a$.

$$
\ln \left[1-F\left(t_{i}\right)\right]=-\lambda t_{i}
$$

By the formula (6) shows that if the statistical fault data to meet the exponential distribution, which is configured to be a linear function through the origin, that is $b=0$. Obviously, due to a fault statistics are random, can not guarantee a straight line through the origin just constructed, this time can be constructed of linear equations to be amended. If the curve does not pass through the origin, we need to fix. To make the fitted curve passes through the origin, a rotation angle at this time can, usually, if an exponential distribution statistics, the value b is small, then a correction value calculation by equation (7).

$$
\tilde{a}=a+b /(T / 2)
$$

Weibull distribution. Cumulative distribution expression two-parameter Weibull distribution like formula (8).

$$
F\left(t_{i}\right)=1-\exp \left[-\left(t_{i} / \eta\right)^{m}\right]
$$

The formula (8) on both sides of the two logarithmic, if Weibull distribution is a linear relationship can be determined by least square method estimates the Weibull distribution parameters, such as the formula (9).

$$
\left\{\begin{array}{l}
\hat{m}=b \\
\hat{\eta}=\exp \left(-\frac{a}{b}\right)
\end{array}\right.
$$

Goodness of fit test.If you find fault scatterplot distribution function in line with the distribution of two or more, you need to determine which distribution and statistical data more in line, then you can compare the goodness of fit. Goodness of fit with R values to represent, (10) as shown in the formula.

$$
R^{2}=\frac{\sum_{i=1}^{n}\left(y_{i}-\bar{y}\right)^{2}}{\sum_{i=1}^{n}\left(\hat{y}_{i}-\bar{y}\right)^{2}}
$$

\section{Reliability Assessment}

Objective of product fault probability density parameter estimation and curve fitting was to assess the reliability index MTBF, which is defined as the formula of formula (11).

$$
M T B F=\int_{0}^{\infty} t f(t) d t
$$

The reliability of index and Weibull can solved by the formula of (12) and (13).

$$
\begin{aligned}
& \text { MTBF }=\frac{1}{\lambda} \\
& M T B F=\eta \gamma \Gamma\left(1+\frac{1}{m}\right)
\end{aligned}
$$




\section{Examples}

To illustrate the electromechanical product fault probability density function estimation and reliability means that a particular calculation standard calculation, is to be calculated as an example of CNC crankshaft grinder. Seven sets of a CNC crankshaft grinder for a period of 4000h fault statistics, a total of 33 statistics to malfunction. The fault data is divided into eight groups, after the failure of data packets, as shown in Table 2.

To determine the distribution type.According to equation (2) can be obtained within a time interval of each faults frequency values of $P\left(t_{i}\right)$, observations probability density value $\hat{f}\left(t_{i}\right)$ and cumulative probability density value $\hat{F}\left(t_{i}\right)$, as shown in Table 3 .

As can be seen from Table 3, the fault probability density of the CNC crankshaft grinder monotonically decreasing trend can be approximately considered to meet the exponential distribution or Weibull distribution.

Tab.2 Grouping and Counting of Fault Data

\begin{tabular}{ccccc}
\hline No. & $t_{i-} / h$ & $t_{i+} / h$ & $t_{i} / h$ & $n_{i}$ \\
\hline 1 & 0 & 500 & 250 & 13 \\
2 & 500 & 1000 & 750 & 7 \\
3 & 1000 & 1500 & 1250 & 4 \\
4 & 1500 & 2000 & 1750 & 3 \\
5 & 2000 & 2500 & 2250 & 2 \\
6 & 2500 & 3000 & 2750 & 2 \\
7 & 3000 & 3500 & 3250 & 1 \\
8 & 3500 & 4000 & 3750 & 1
\end{tabular}

Tab.3 Fault Frequency, Probability Density and Accumulative Probability Density

\begin{tabular}{cccc}
\hline$t_{i} / h$ & $P\left(t_{i}\right)$ & $\hat{f}\left(t_{i}\right)$ & $\hat{F}\left(t_{i}\right)$ \\
\hline 250 & 0.393939 & 0.000788 & 0.393939 \\
750 & 0.212121 & 0.000424 & 0.606061 \\
1250 & 0.121212 & 0.000242 & 0.727273 \\
1750 & 0.090909 & 0.000182 & 0.818182 \\
2250 & 0.060606 & 0.000121 & 0.878788 \\
2750 & 0.060606 & 0.000121 & 0.939394 \\
3250 & 0.030303 & 0.000061 & 0.969697 \\
3750 & 0.030303 & 0.000061 & 1.000000 \\
\hline
\end{tabular}

Parameter Estimation.The following estimate parameters to exponential and Weibull distribution.

Exponential Distribution. By the formula (4) and (6) and Table 3 data, the method of least squares linear regression, linear function obtained as follows:

$$
y=-9.67 \times 10^{-4} x-0.143
$$

Fitting a curve shown in Figure 2 to achieve. As can be seen from the figure, the curve does not fit through the origin, the need for correction by equation (10) is a linear function of the corrected:

$$
y=-1.14 \times 10^{-3}
$$

It is possible to obtain the modified exponential distribution parameters of $\lambda$ valuation point:

$$
\lambda=1.14 \times 10^{-3}
$$




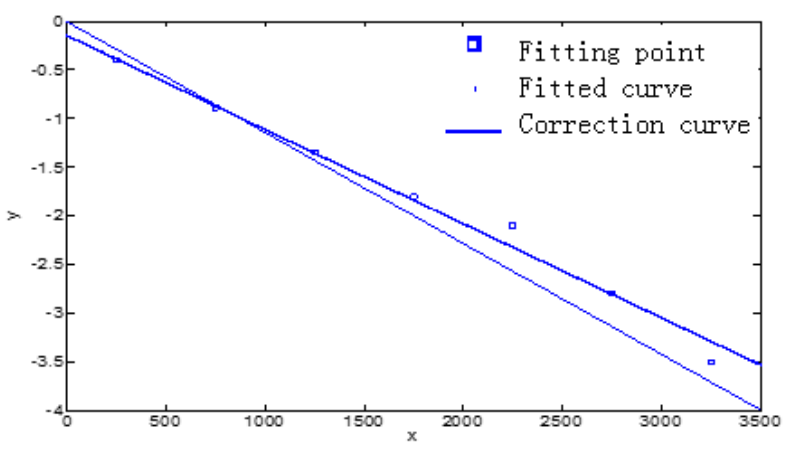

Fig.1 Fitting of Exponential Distribution

Weibull distribution.By the formula (4) and (8) and Table 3 data, the method of least squares linear regression, linear function obtained as follows:

$$
y=0.737 x-4.873
$$

Fitting a curve shown in Figure 3. By the formula (9) can be drawn point Weibull distribution parameters $m$ and $\eta_{\text {are estimated values are: }}$

$$
\left\{\begin{array}{l}
\hat{m}=-4.873 \\
\hat{\eta}=1.163
\end{array}\right.
$$

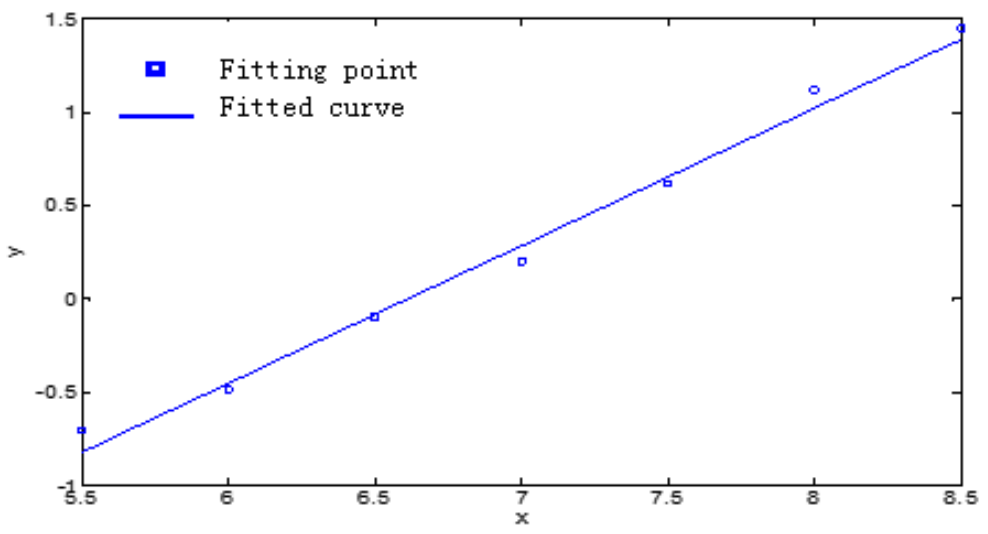

Fig.2 Fitting of Weibull Distribution

Goodness of fit test.To determine which profile is more consistent with the statistical data, can goodness of fit test. The use of formula (10) were calculated goodness of fit goodness of fit exponential distribution and Weibull distribution of $R_{e}$ and $R_{W}$ is:

$$
\left\{\begin{array}{l}
R_{e}=0.873 \\
R_{\omega}=0.793
\end{array}\right.
$$

From the goodness of fit value can also determine the effect of the exponential distribution fits better than the Weibull distribution, namely that the probability of fault of CNC crankshaft grinder distribution exponential distribution.

Reliability exponential calculation.In this example, follow the exponential distribution, using equation (12) can be calculated reliability indices CNC crankshaft grinding machine as follows:

$$
M T B F=\frac{1}{\lambda}=877 h
$$

If the direct MTBF by definition, you can calculate the sample machine reliability indicators: 


$$
\text { MTBF }=\frac{n t_{\text {test }}}{n_{t}}=\frac{7 \times 4000}{33}=848 \mathrm{~h}
$$

As can be seen, the reliability exponential of the fault distribution function obtained directly calculate the sample machine reliability indices are close, but there are errors, mainly censored time long enough, with the increase censored time, fault number showing a decreasing trend, it will increase MTBF.

\section{Summary}

(1)Analysis using the least squares method for failure probability density function parameter estimation of CNC machine tools common type of fault probability distribution is proposed to determine the type of electromechanical product fault distribution steps and methods are introduced. The proposed method can easily write a computer program to estimate the parameters and reliability index calculation.

(2) For example of CNC crankshaft grinder, for a period of 4000h fault statistics, fault data were grouped CNC crankshaft grinder probability density parameter estimation and fitting, exponential and Weibull were goodness of fit test, and calculate the reliability index of CNC crankshaft grinder.

(3)Using reliability Assessment Method of the proposed use and reliability of the direct use of the definition is closer to solving MTBF reliability evaluation method proposed to evaluate the reliability of mechanical and electrical products, designed to provide a reference for reliability.

\section{References}

[1] B.Samanta, B.Sarkar, S.K.Mukherjee.Reliability assessment of hydraulic shovel system using fault trees $[\mathrm{J}]$.Transactions of the Institutions of Mining and Metallurgy, Section A:Mining Technology, 2002 (111) : 129-135.

[2]Yang Na, Ye Guo-ming.Summary of qualitative and quantitative design methods on machinery reliability [J] .Machinery Design \& Manufacture, 2006 (3) : 16-19.

[3] L.Goel, X.Liang, Y.Ou.Monte Carlo simulation -based customer service reliability assessment

[J] .Electric Power Systems Research, 1999, 49 (3) : 185-194.

[4] Feng Chang -you, Wang Xi -fan, Bie Zhao -hong.Unit maintenance scheduling model based on system reliability [J]. Journal of Xi'an Jiaotong University, 2009, 43 (8) : 80-85.

[5] C.Y.Li, M.Q.Xu, S.Guo.Real-time reliability assessment based on gamma process and bayesian estimation [J].Journal of Astronautics, 2009, 30 (4) : 1722-1726.

[6] Xu Ge-ning, Li Yin-de, Yang Heng.Reliability assessment of hydraulic systems in automobile crane based on Bayesian networks [J] .China Safety Science Journal, 2011，21 (5) : 90-96). [7] G.Yuriy, N.Vayenas.Discrete-event simulation of mine equipment systems combined with a reliability assessment model based on genetic algorithms [ J ] .International Journal of Mining,

Reclamation and Environment, 2008, 22 (1) : 70-83.

[8] Xue Yu-xia.Research on availability key technique for numerical control machine tool [D] .Changchun: Jilin University, 2009..

[9] S.C.Kang, H.M.Koh, J.F.Choo.An efficient response surface method using moving least squares approximation for structural reliability analysis $[\mathrm{J}]$.Probabilistic Engineering Mechanics, 2010,25 (4) : 365-371. 